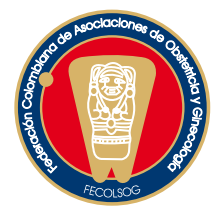

SERIE De CASOS

\title{
ENFERMEDADES GLOMERULARES DURANTE LA GESTACIÓN. SERIE DE CASOS Y REVISIÓN DE LA LITERATURA
}

\author{
Glomerular disease and pregnancy \\ Jorge M. Gómez-Jiménez, M.D. *, Luis F. Arias, M.D., Ph.D.** \\ Recibido: agosto 15/08 - Aceptado: noviembre 25/08
}

\section{RESUMEN}

Objetivos: determinar las glomerulopatías más frecuentes y su curso clínico en mujeres gestantes. Hacer una revisión de la literatura del tema.

Materiales y métodos: serie de casos retrospectivos de pacientes gestantes con enfermedad glomerular activa diagnosticada por biopsia, entre enero de 2000 y septiembre de 2007, atendidas en el Hospital San Vicente de Paúl de Medellín, Colombia. No hubo exclusiones. Se determinaron sus características clínicas, la evolución de la enfermedad glomerular y el resultado materno perinatal.

Resultados: se hizo el diagnóstico en 11 pacientes. Cuatro casos de nefritis lúpica, dos de glomerulonefritis rápidamente progresiva (GNRP) por GN extracapilar y granulomatosis de Wegener, respectivamente; tres con síndrome nefrótico por glomeruloesclerosis focal y segmentaria (GEFyS), una con GN membranoproliferativa tipo I, y una con GN proliferativa mediada por complejos inmunes. En dos casos se presentó eclampsia. En tres casos hubo muerte materna: dos por eclampsia y una por granulomatosis de Wegener; y en tres casos muerte fetal: dos por eclampsia y una en materna con nefritis lúpica y síndrome antifosfolípido. Las

* Departamento de Ginecología y Obstetricia, y grupo PRYT; Departamento de Patología, Facultad de Medicina, Universidad de Antioquia, y Hospital San Vicente de Paúl, Medellín, Colombia.

** Grupo PRYT; Departamento de Patología, Facultad de Medicina, Universidad de Antioquia, y Hospital San Vicente de Paúl, Medellín, Colombia. Correo electrónico: luisfer_uda@yahoo.com glomerulopatías primarias no mostraron empeoramiento durante la gestación.

Conclusiones: en casos de GN durante la gestación hay riesgo incrementado de complicaciones materno-fetales.

Palabras clave: biopsia renal, embarazo, enfermedad glomerular, gestación, glomerulopatías, hispánicas.

\section{SUMMARY}

Aims: determining the most frequent glomerulopathies in cases of biopsy-proved glomerular disease which was active during pregnancy; the literature was searched for such patients. Ascertaining clinical-pathological features, glomerular disease evolution, and maternal and obstetrical outcomes.

Material and methods: this was a descriptive, retrospective study of a case series. Our archives were searched (01/2000 to 09/2007) for all cases of pregnant women who attended the Hospital San Vicente de Paúl, Medellín, Colombia, with biopsy-proved glomerular disease. There were no exclusions. Maternal and neonatal complications were studied.

Results: eleven patients constituted our study: four cases of lupus nephritis, two rapidly progressive glomerulonephritis (RPGN) due to crescentic GN and Wegener's granulomatosis, three nephrotic syndrome cases due to focal and segmental glomerulosclerosis (FSGS), one 
case with type I membranoproliferative GN and another one with immune complex-mediated proliferative GN. There were eclampsia and "endotheliosis" in two cases. Maternal outcome was fatal in three cases: two eclamptic women and another one with Wegener's granulomatosis. Three stillbirths occurred in our 11 cases, two in eclamptic patients and another one in a woman with lupus nephritis and antiphospholipid syndrome. Primary glomerulopathies did not demonstrate impairment during pregnancy.

Conclusions: pregnant women suffering from glomerular disease have an increased risk of maternal and foetal complications occurring; however, maternal and foetal outcomes can be improved with intensive medical care. Rigorous obstetric control is necessary in pregnant women with lupus nephritis. Renal biopsy may be postponed until after giving birth in cases where there is no precise indication for treatment.

Key words: glomerular disease, glomerulopathy, glomerulonephritis, Hispanics, pregnancy, renal biopsy.

\section{INTRODUCCIÓN}

En las dos décadas pasadas surgieron importantes publicaciones sobre el curso de las glomerulonefritis $y$, en general, de las enfermedades renales durante la gestación. ${ }^{1-10}$ Estos trabajos permitieron afirmar que el impacto en los resultados de la gestación no es muy ominoso, excepto cuando se presenta hipertensión severa pregestacional y/o preeclampsia, o anticuerpos antifosfolípido. Estas conclusiones se han generalizado para enfermedades glomerulares secundarias y primarias. ${ }^{11}$

En pacientes obstétricas con enfermedad renal, y más específicamente glomerular, hay aspectos que desconocemos en nuestro medio, tales como: la frecuencia, el manejo de las complicaciones y el pronóstico; por lo tanto, el objetivo del presente trabajo fue determinar la frecuencia de la enfermedad glomerular en el embarazo, sus características clínicas, sus complicaciones y el pronóstico materno-fetal en un grupo de pacientes de Colombia, y hacer una revisión de la literatura pertinente.

\section{MATERIALES Y MÉTODOS}

Diseño: serie de casos.

Población: se incluyeron todos los casos de pacientes con glomerulopatía confirmada por biopsia y enfermedad activa durante la gestación, atendidas en el período comprendido desde enero de 2000 hasta septiembre de 2007, en el Hospital San Vicente de Paúl de Medellín, Colombia; hospital general de referencia de la región noroccidental del país, que atiende pacientes de la seguridad social privada y pública. No hubo exclusiones. Muestreo secuencial por conveniencia.

Procedimiento: a partir de los archivos de biopsias renales, se ubicaron los casos de pacientes con diagnóstico de enfermedad glomerular durante el embarazo. Se tomó la información de la historia clínica. Todas las biopsias fueron revisadas y el diagnóstico reconfirmado. Para el diagnóstico de estas biopsias dispusimos del estudio histopatológico e inmunopatológico (inmunofluorescencia directa para $\operatorname{IgA}, \operatorname{IgG}$, IgM, C3, C1q, y cadenas ligeras kappa y lambda). Se describen los hallazgos clinicopatológicos, el tratamiento, la evolución de las pacientes y del producto de la concepción.

Los datos se presentan mediante estadísticas descriptivas y se presenta la proporción de enfermedad glomerular en la gestación, en relación a las biopsias renales tomadas en el período de estudio.

\section{RESULTADOS}

Entre 837 biopsias de riñón nativo con glomerulopatías (primarias y secundarias) durante el período de tiempo indicado, detectamos 11 casos $(1,3 \%)$ de enfermedad glomerular en la gestación. Los datos demográficos, características clínicas, diagnóstico anatomopatológico de base, y evolución materna y fetal, aparecen en la tabla 1. 


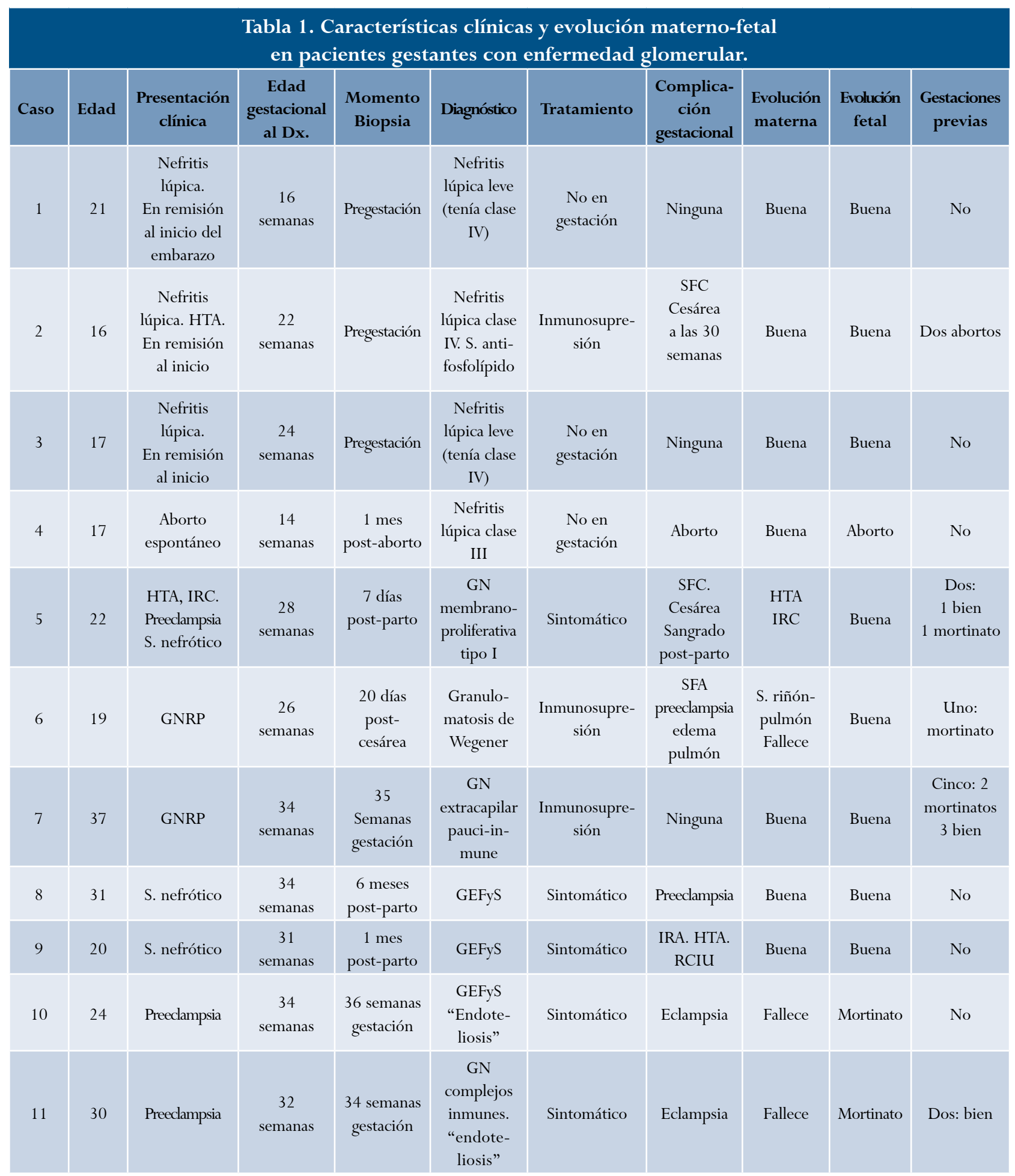

HTA: hipertensión arterial sistémica; IRC: insuficiencia renal crónica; IRA: insuficiencia renal aguda; GN: glomerulonefritis; GNRP: GN rápidamente progresiva; GEFyS: glomeruloesclerosis focal y segmentaria; SFA: sufrimiento fetal agudo; SFC: sufrimiento fetal crónico; RCIU: retardo del crecimiento intrauterino; S.: síndrome.

\section{DISCUSIÓN}

Presentamos una pequeña serie de casos con enfermedad renal glomerular durante la gestación, confirmada por estudio anatomopatológico, haciendo énfasis en el resultado materno y fetal. La glomerulopatía más frecuente en nuestros casos es nefritis lúpica, algo que no es de extrañar debido a que el LES suele afectar con mayor frecuencia 
a mujeres en edad fértil. Es conocido que el LES afecta especialmente a las mujeres entre los 10 y 40 años y es más frecuente en mujeres de raza negra, indígenas y asiáticas. ${ }^{12,13}$ En nuestra población no gestante, la glomerulopatía primaria más frecuente es la glomeruloesclerosis focal y segmentaria. ${ }^{14}$ En mujeres hispanoamericanas existe más frecuente y severo compromiso renal que en otros grupos étnicos. ${ }^{15,16}$

Las enfermedades glomerulares pueden estar presentes antes o desarrollarse durante el embarazo. Las glomerulopatías primarias más frecuentes durante la gestación son GEFyS, GN membranosa, nefropatía IgA, GN membranoproliferativa y GN extracapilar (“crescentic"). ${ }^{2,5,6}$ Se ha descrito que el embarazo en las pacientes con nefritis lúpica puede cursar con mayor frecuencia de pérdidas fetales, con mayor riesgo de exacerbaciones renales, de deterioro renal, de preeclampsia severa y temprana, y parto prematuro, especialmente cuando no han transcurrido seis meses después de la remisión, y si se presenta hipertensión pregestacional, SAF, anticuerpos anticardiolipina o anticoagulante lúpico. ${ }^{17-24}$ La gestación en mujeres con nefritis lúpica es exitosa en un porcentaje muy variable (20-90\%) de acuerdo, principalmente, con la función renal. ${ }^{22}$ Según A. Tandon, et al, ${ }^{25}$ la nefritis lúpica no es exacerbada por la gestación.

No hay indicación absoluta de biopsia renal durante la gestación. Sin embargo, debe realizarse para distinguir y clasificar nefropatías graves. Cuando hay deterioro rápido de la función renal, puede requerirse la terminación de la gestación. La biopsia renal es un procedimiento con morbilidad, que debería considerarse sólo si ofrece a la paciente la oportunidad de un mejor manejo. ${ }^{26}$ En casos de síndrome nefrítico y GNRP puede determinar el tratamiento más óptimo y oportuno, como quedó evidenciado en el caso 7, en el que el diagnóstico de la biopsia renal permitió el tratamiento más adecuado. En síndrome nefrótico, es necesario sopesar la necesidad de un tratamiento agresivo rápido o esperar la terminación del embarazo, para realizar la biopsia e iniciar un tratamiento (usualmente inmunosupresor). ${ }^{26-28}$

Las mujeres con IRC no deberían embarazarse. Si así fuere, siempre se considerará como una gestación de alto riesgo obstétrico. El pronóstico materno y fetal empeora de acuerdo con la severidad de la IRC. En cerca de la mitad de las pacientes con insuficiencia renal crónica moderada o severa se presentan complicaciones obstétricas, entre ellas el síndrome HELLP. ${ }^{29}$

Sólo hay una enfermedad glomerular exclusiva del embarazo y es la "glomerulopatía preeclámptica" (usualmente se le da el término impreciso de "endoteliosis"). ${ }^{26}$

Muchos estudios han evaluado si el embarazo afecta adversamente el curso natural de las enfermedades glomerulares y si la evolución del embarazo está influenciada por la glomerulopatía; ;-4,6,7,30 sin embargo, hay pocos estudios controlados que permitan sacar conclusiones claras. Datos experimentales sugieren efectos adversos de la hemodinámica "anormal" de la gestación. ${ }^{31}$ En un estudio por Jungers, et al, ${ }^{32}$ el riesgo de desarrollar IRC no se incrementó en 148 mujeres gestantes con varias enfermedades renales comprobadas con biopsia, comparadas con un grupo control de mujeres con enfermedades similares, pero no gestantes. Suele haber un empeoramiento transitorio de la hipertensión, la proteinuria y la disfunción renal en pacientes con glomerulopatías preexistentes. ${ }^{2,32}$ En una revisión por C. Baylis, ${ }^{33}$ el autor dice que en pacientes con buena filtración glomerular antes de la concepción, usualmente no hay efectos del embarazo en la enfermedad renal. Hipertensión no controlada, proteinuria en rango nefrótico o alteración de la función renal al momento de la concepción o en fases iniciales de la gestación, están asociadas con riesgo aumentado de deterioro de la función. ${ }^{2,4,6,7,10,31,34,35}$

En pacientes con GN membranosa, nefropatía IgA y GN proliferativa, la incidencia de parto normal está entre el 71 y el 80\%., ${ }^{9,36}$ En GEFyS hay mayor incidencia de complicaciones maternas y fetales, 
con un peor pronóstico que GN membranosa y nefropatía IgA. 5,6,8,34,37,38 En granulomatosis de Wegener y GN extracapilar, también se informa riesgo aumentado de recaídas de la enfermedad renal y mayor riesgo de complicaciones y muertes fetales. ${ }^{39-43}$ Además de pérdida fetal, se informan como complicaciones el parto prematuro y el retardo del crecimiento intrauterino. En el conjunto de GN se informa, aproximadamente, $10-15 \%$ de pérdidas fetales. ${ }^{31}$

Finalmente, es importante al analizar en la literatura los resultados de las series más grandes de pacientes, tener en cuenta que muchas de ellas son trabajos de los años 80 e incluyen pacientes con gestaciones durante los años 60, 70 y 80 . Las condiciones de diagnóstico y tratamiento han cambiado, por lo que es probable que el progreso o no de las enfermedades glomerulares (y en general de todas las enfermedades renales) y el resultado materno-fetal sean diferentes (mejores) en la actualidad. ${ }^{35}$ Los casos presentados, aunque no constituyen una serie grande, reflejan la situación en un país de pacientes hispánicas.

\section{REFERENCIAS}

1. Katz AI, Davison JM, Hayslett JP, Singson E, Lindheimer MD. Pregnancy in women with kidney disease. Kidney Int 1980:18:192-206.

2. Surian M, Imbasciati E, Cosci P, Banfi G, Barbiano di Belgiojoso G, Brancaccio D, et al. Glomerular disease and pregnancy. A study of 123 pregnancies in patients with primary and secondary glomerular diseases. Nephron 1984;36:101-5.

3. Rovati C, Perrino ML, Barbiano di Belgiojoso G, Surian M, Radaelli L, Minetti L. Pregnancy and course of primary glomerulonephritis. Contrib Nephrol 1984;37:182-9.

4. Barceló P, López-Lillo J, Cabero L, Del Río G. Successful pregnancy in primary glomerular disease. Kidney Int 1986;30:914-9.

5. Kincaid-Smith P, Fairley KF. Renal disease in pregnancy. Three controversial areas: mesangial IgA nephropathy, focal glomerular sclerosis (focal and segmental hyalinosis and sclerosis), and reflux nephropathy. Am J Kidney Dis 1987;9:328-33.
6. Packham DK, North RA, Fairley KF, Kloss M, Whitworth JA, Kincaid-Smith P. Primary glomerulonephritis and pregnancy. Q J Med 1989;71:537-53.

7. Imbasciati E, Ponticelli C. Pregnancy and renal disease: predictors for fetal and maternal outcome. Am J Nephrol 1991;11:353-62.

8. Jungers P, Houillier P, Forget D, Labrunie M, Skhiri $\mathrm{H}$, Giatras I, et al. Influence of pregnancy on the course of primary chronic glomerulonephritis. Lancet 1995;28:1122-4.

9. Alexopoulos E, Bili H, Tampakoudis P, Economidou D, Sakellariou G, Mantalenakis S, et al. Outcome of pregnancy in women with glomerular diseases. Ren Fail 1996;18:121-9.

10. Holley JL, Bernardini J, Quadri KH, Greenberg A, Laifer SA. Pregnancy outcomes in a prospective matched control study of pregnancy and renal disease. Clin Nephrol 1996;45:77-82.

11. Fischer MJ, Lehnerz SD, Hebert JR, Parikh CR. Kidney disease is an independent risk factor for adverse fetal and maternal outcomes in pregnancy. Am J Kidney Dis 2004;43:415-23

12. Lau CS, Yin G, Mok MY. Ethnic and geographical differences in systemic lupus erythematosus: an overview. Lupus 2006;15:715-9.

13. Patel M, Clarke AM, Bruce IN, Symmons DP. The prevalence and incidence of biopsy-proven lupus nephritis in the UK: evidence of an ethnic gradient. Arthritis Rheum 2006;54:2963-9.

14. Mejía G, Builes M, Arbeláez M, Henao JE, Arango JL, García A. Descripción clínico-patológica de las enfermedades glomerulares. Estudio de 383 biopsias renales. Acta Med Colomb 1989;14:369-74.

15. Tejani A, Nicastri AD, Chen CK, Fikrig S, Gurumurthy K. Lupus nephritis in black and Hispanic children. Am J Dis Child 1983;137:481-3.

16. Calvo-Alén J, Reveille JD, Rodríguez-Valverde V, McGwin G Jr, Baethge BA, Friedman AW, et al. Clinical, immunogenetic and outcome features of Hispanic systemic lupus erythematosus patients of different ethnic ancestry. Lupus 2003;12:377-85.

17. Packham DK, Lam SS, Nicholls K, Fairley KF, KincaidSmith PS. Lupus nephritis and pregnancy. Q J Med 1992;83:315-24.

18. Le Thi Huong D, Wechsler B, Piette JC, Bletry O, Godeau P. Pregnancy and its outcome in systemic lupus erythematosus. QJM 1994;87:721-9. 
19. Le Thi Huong D, Wechsler B, Vauthier-Brouzes D, Beaufils H, Lefebvre G, Piette JC. Pregnancy in past or present lupus nephritis: a study of 32 pregnancies from a single centre. Ann Rheum Dis 2001;60:599-604.

20. Moroni G, Ponticelli C. Pregnancy after lupus nephritis. Lupus 2005;14:89-94.

21. Carmona F, Font J, Moga I, Làzaro I, Cervera R, Pac $\mathrm{V}$, et al. Class III-IV proliferative lupus nephritis and pregnancy: a study of 42 cases. Am J Reprod Immunol 2005;53:182-8.

22. Germain S, Nelson-Piercy C. Lupus nephritis and renal disease in pregnancy. Lupus 2006;15:148-55.

23. Molad Y. Systemic lupus erythematosus and pregnancy. Curr Opin Obstet Gynecol 2006;18:613-7.

24. Whitelaw DA, Hall D, Kotze T. Pregnancy in systemic lupus erythematosus: a retrospective study from a developing community. Clin Rheumatol 2008:27:577-80.

25. Tandon A, Ibáñez D, Gladman DD, Urowitz MB. The effect of pregnancy on lupus nephritis. Arthritis Rheum 2004;50:3941-6.

26. Kuller JA, D'Andrea NM, McMahon MJ. Renal biopsy and pregnancy. Am J Obstet Gynecol 2001;184:1093-6.

27. Pandya BK, Gibson SP, Robertson IG. Nephrotic syndrome in early pregnancy--is renal biopsy always necessary? Nephrol Dial Transplant 2002;17:672-4.

28. Day C, Hewins P, Hildebrand S, Sheikh L, Taylor G, Kilby M, et al. The role of renal biopsy in women with kidney disease identified in pregnancy. Nephrol Dial Transplant 2008;23:201-6.

29. Jones DC, Hayslett JP. Outcome of pregnancy in women with moderate or severe renal insufficiency. N Engl J Med 1996;335:226-32.

30. Cameron JS, Hicks J. Pregnancy in patients with pre-existing glomerular disease. Contrib Nephrol 1984;37:149-56.

31. Jungers P, Chauveau D. Pregnancy in renal disease. Kidney Int 1997;52:871-85.

32. Jungers P, Houillier P, Forget D, Henry-Amar M. Specific controversies concerning the natural history of renal disease in pregnancy. Am J Kidney Dis 1991;17:116-22.

33. Baylis C. Impact of pregnancy on underlying renal disease. Adv Ren Replace Ther 2003;10:31-9.

34. Hemmelder MH, de Zeeuw D, Fidler V, de Jong PE. Proteinuria: a risk factor for pregnancy-related renal function decline in primary glomerular disease? Am J Kidney Dis 1995:26:187-92.

35. Bar J, Orvieto R, Shalev Y, Peled Y, Pardo Y, Gafter $\mathrm{U}$, et al. Pregnancy outcome in women with primary renal disease. Isr Med Assoc J 2000;2:178-81.

36. Abe S, Amagasaki Y, Konishi K, Kato E, Sakaguchi H, Iyori S: The influence of antecedent renal disease on pregnancy. Am J Obstet Gynecol 1985;153:508-14.

37. Jungers P, Forget D, Houillier P, Henry-Amar M, Grünfeld JP. Pregnancy in IgA nephropathy, reflux nephropathy, and focal glomerular sclerosis. Am J Kidney Dis 1987;9:334-8.

38. Packham DK, North RA, Fairley KF, Ihle BU, Whitworth JA, Kincaid-Smith P. Pregnancy in women with primary focal and segmental hyalinosis and sclerosis. Clin Nephrol 1988;29:185-92.

39. Habib A, MacKay K, Abrons HL. Wegener's granulomatosis complicating pregnancy: presentation of two patients and review of the literature. Clin Nephrol 1996;46:332-6.

40. Luisiri P, Lance NJ, Curran JJ. Wegener's granulomatosis in pregnancy. Arthritis Rheum 1997;40:1354-60.

41. Harber MA, Tso A, Taheri S, Tuck SM, Burns A. Wegener's granulomatosis in pregnancy--the therapeutic dilemma. Nephrol Dial Transplant 1999;14:1789-91.

42. Piccoli GB, Mezza E, Bontempo S, Burdese M, Soragna G, Gai M, et al. Vasculitis and kidney involvement in pregnancy: evidence-based medicine and ethics bear upon clinical choices. Nephrol Dial Transplant 2004;19:2909-13.

43. Seo P. Pregnancy and vasculitis. Rheum Dis Clin North Am 2007;33:299-317. 